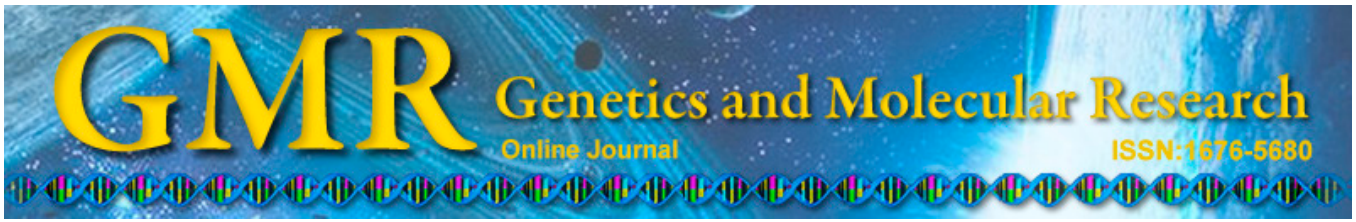

\title{
Genetic diversity and classification of Tibetan yak populations based on the mtDNA COIII gene
}

\author{
Q.Q. Song ${ }^{1}$, Z.X. Chai ${ }^{1}$, J.W. Xin ${ }^{2}$, S.J. Zhao ${ }^{1}$, Q.M. Ji' ${ }^{2}$ C.F. Zhang', \\ Z.J. Ma ${ }^{3}$ and J.C. Zhong ${ }^{1}$
}

${ }^{1}$ Key Laboratory of Animal Genetics and Breeding, State Ethnic Affairs Commission and Ministry of Education, Southwest University for Nationalities, Chengdu, China ${ }^{2}$ Institute of Animal Science and Veterinary, Tibet Academy of Agricultural and Animal Husbandry Sciences, Lhasa, China ${ }^{3}$ Institute of Animal Science,

Qinghai Academy of Animal Science and Veterinary Medicine, Xining, China

Corresponding author: J.C. Zhong

E-mail: zhongjincheng518@126.com

Genet. Mol. Res. 14 (1): 1763-1770 (2015)

Received February 10, 2014

Accepted July 23, 2014

Published March 13, 2015

DOI http://dx.doi.org/10.4238/2015.March.13.3

\begin{abstract}
To determine the level of genetic diversity and phylogenetic relationships among Tibetan yak populations, the mitochondrial DNA cytochrome c oxidase subunit 3 (COIII) genes of 378 yak individuals from 16 populations were analyzed in this study. The results showed that the length of cytochrome c oxidase subunit 3 gene sequences was $781 \mathrm{bp}$, with nucleotide frequencies of $29.2,29.4,26.1$, and $15.2 \%$ for T, C, A, and G, respectively. A total of 26 haplotypes were identified, with 69 polymorphic sites, including 11 parsimony-informative sites and 58 singlenucleotide polymorphism sites. No deletions/insertions were found in sequence comparison, indicating that nucleotide mutation types were transitions and transversions. Haplotype and nucleotide
\end{abstract}


diversities were 0.562 and 0.00138 , respectively, indicating a high level of genetic diversity in Tibetan yak populations. Phylogenetic relationship analysis indicated that Tibetan yak populations are divided into 2 groups.

Key words: Mitochondrial DNA cytochrome c oxidase subunit 3; Genetic diversity; Phylogenetic relationship; Tibetan yak

\section{INTRODUCTION}

The yak (Bos grunniens) is currently regarded as an important renewable genetic resource, as it thrives in extremely harsh environments at high altitudes, hypoxia, severely cold winters, and cool moist summers; the yak survives short growing seasons with limited grazing resources (Wiener et al., 2003). As a unique species on the Qinghai-Tibetan Plateau and in adjacent mountains and subalpine regions, the yak is a multi-purpose domestic animal utilized by local people. More than 4.9 million domestic yaks live in Tibet of China, accounting for approximately $30 \%$ of all Chinese domestic yaks (Zhang, 2012).

Mitochondrial DNA (mtDNA) is a self-replicating, maternally inherited, circular DNA molecule that has been widely used to resolve taxonomic controversies (Delarbre et al., 1998; Boore et al., 1998; Lavrov and Lang, 2005; Gissi et al., 2008; Mipam et al., 2012). It is composed of 13 protein subunits, 22 mitochondrial tRNAs, 2 mitochondrial-specific ribosomal RNAs, and 12S and 16S rRNAs (Delarbre et al., 1998; Tiranti et al., 2000). The COIII gene is 1 of 13 protein-coding genes in the mitochondrial genome. Cloning and sequence analysis of the COIII gene are very valuable for studying molecular system evolution and species classification. Recent studies have examined the COIII gene in several species, including fish, frogs, mammals, birds (Griffiths et al., 1998), mosquitos (Pridgeon et al., 2009), and rats (Huang et al., 2004). In addition, previous studies have been performed on several mtDNA genetic markers in Tibetan yak populations (Zhao et al., 2011; Chai et al., 2011; Ji et al., 2012; Zhang et al., 2012). However, the COIII gene has not been investigated in Tibet yak populations. In this study, we sequenced the complete COIII gene in 378 individuals representing 16 Tibetan yak populations, including the Sibu, Riduo, Leiwuqi, Dingqing, Jiangda, Sangri, Cuona, Longzi, Pali, Sangsang, Kangbu, Zhongba, Jiali, Baqing, Nierong, and Gongbujiangda yak populations. The aim of this study was to evaluate the genetic diversity of Tibetan yak populations and explore their classification relationships.

\section{MATERIAL AND METHODS}

\section{Sample collection and DNA extraction}

A total of 378 ear tissue samples were collected from 16 healthy yak populations in Tibet. Details regarding sample number, name, and geographical distribution of the populations are summarized in Table 1. After collection, the samples dipped in $75 \%$ ethyl alcohol were transported to the lab and stored at $-80^{\circ} \mathrm{C}$. A DNA Extraction Kit (Tiangen, Beijing, China) was used to extract genomic DNA, which was detected on a $1 \%$ agarose gel and stored at $-20^{\circ} \mathrm{C}$ until use. 
Table 1. Distribution area, code, number, and sampling locations of samples.

\begin{tabular}{lllcl}
\hline Distribution area & Population & Code & Number of samples & Sampling locations \\
\hline Lasa & Sibu yak & SB & 10 & Lasa \\
& Riduo yak & RD & 51 & Riduo village \\
Changdu & Leiwuqi yak & LW & 25 & Leiwuqi county \\
& Dingqing yak & DQ & 50 & Dingqing county \\
\multirow{5}{*}{ Shannan } & Jiangda yak & JD & 10 & Jiangda county \\
& Sangri yak & SR & 10 & Cuonga county \\
& Cuona yak & CN & 26 & Longzi county \\
Rikaze & Longzi yak & LZ & 49 & Pali town \\
& Pali yak & PL & 10 & Sangsang town \\
& Sangsang yak & SS & 10 & Kangbu village \\
& Kangbu yak & KB & 10 & Zhongba county \\
Naqu & Zhongba yak & ZB & 36 & Jiali county \\
& Jiali yak & JL & 10 & Baqing county \\
& Baqing yak & BQ & 11 & Nierong county \\
Linzhi & Nierong yak & NR & 50 & Gongbujiangda county \\
\hline
\end{tabular}

\section{PCR amplification and sequencing}

The COIII gene in each sample was amplified by polymerase chain reaction (PCR) using the primers PF 5'-TGTGAGCAGGAGCCGTAA-3' and PR 5'-CCATATTCGGTTCATTCC AGTC-3'. PCR amplifications were carried out in a $50-\mu \mathrm{L}$ reaction tube containing $200 \mathrm{ng}$ genomic DNA, 20 pmol of each primer, $25 \mu \mathrm{L} 2 \mathrm{X}$ long Taq premixed DNA polymerase (TaKaRa, Dalian, China), and $19 \mu \mathrm{L}$ sterilized $\mathrm{ddH}_{2} \mathrm{O}$. PCR amplifications were performed as follows: initial denaturation step at $95^{\circ} \mathrm{C}$ for $2 \mathrm{~min}$, followed by 35 cycles at $95^{\circ} \mathrm{C}$ for $30 \mathrm{~s}$, $57.3^{\circ} \mathrm{C}$ for $30 \mathrm{~s}, 72^{\circ} \mathrm{C}$ for $1 \mathrm{~min}$, and a final extension step at $72^{\circ} \mathrm{C}$ for $7 \mathrm{~min}$. PCR products were purified using the DNA Gel Extraction Kit (Tiangen) according to manufacturer instructions and sequenced on an ABI 3730xl automated sequencer (Invitrogen, Carlsbad, CA, USA).

\section{Data analyses}

mtDNA COIII sequences were compiled using the SeqManII software (DNASTAR, Inc., Madison, WI, USA) and aligned using ClustalW 1.83 (Thompson et al., 1997) with default parameters. Nucleotide polymorphisms within the sequences were computed using MEGA 4.0 (Tamura et al., 2007). Genetic distances between populations were calculated using Kimura double parameters. A phylogenetic tree was constructed based on the Kimura's 2-parameter model using the MEGA 4.0 software with the neighbor-joining method. The confidence level of individual nodes of the phylogenetic tree was assessed using bootstrap resampling analysis (1000 replicates). Polymorphic sites, haplotype diversity, and nucleotide diversity were analyzed using DnaSP 4.0 (Rozas et al., 2003).

\section{RESULTS}

\section{Genetic diversity among populations}

Tibetan yak mtDNA COIII gene sequences were successfully obtained. The length of yak COIII sequences was 781 bp and the average ratios of T, C, A, and G were $29.2 \%$ (29.1$29.6 \%), 29.4 \%$ (29.1-29.6\%), 26.1\% (25.9-26.6\%), and 15.2\% (14.9-15.5\%), respectively. 
A total of 69 polymorphic sites were detected in the sequence comparisons of the Tibetan yak mtDNA COIII gene, including 11 parsimony-informative sites and 58 nucleotide polymorphism sites. No deletions or insertions were found in sequence comparison, indicating that nucleotide mutations included only transitions and transversions. The transition/transversion ratio was 12.89. Most base substitutions were T/C transitions, accounting for approximately $57 \%$ of all nucleotide mutations. The frequency of transformations was significantly higher than that of transversions.

Additionally, 26 haplotypes were identified in the study, with an average of 3.75 (Table 2). Haplotype diversity ranged from $0.315-0.844$, with an average of 0.562 (Table 3 ), with the highest level of genetic diversity within the Sibu yak population and the lowest level of genetic diversity in the Riduo yak population. Nucleotide diversity ranged from 0.00051-0.00347, with an average of 0.00138 , with the highest level of genetic diversity in Dingqing yak and the lowest level of genetic diversity in Jiali yaks. Haplotype and nucleotide diversities of Sibu, Gongbujiangda, Leiwuqi, Sangri, Longzi, and Jiangda yaks were relatively higher than in other populations. The haplotype and nucleotide diversities of Baqing, Jiali, Kangbu, Sangsang, Cuona, Nierong, Riduo, and Zhongba yaks were relatively lower than in other populations.

Table 2. Distribution of haplotypes of Tibetan yak populations.

\begin{tabular}{|c|c|c|}
\hline Haplotype & Number of haplotypes & Distribution of haplotypes \\
\hline Hap-1 & 45 & DQ (6), LW (6), LZ (9), NR (8), RD (3), ZB (5), BQ (2), GD (2), SR (4) \\
\hline Hap-2 & 1 & $\mathrm{JD}(1)$ \\
\hline Hap-3 & 1 & SS (1) \\
\hline Hap-4 & 60 & $\begin{array}{l}\mathrm{CN}(7), \mathrm{DQ}(10), \mathrm{LW}(3), \mathrm{LZ}(6), \mathrm{NR}(7), \mathrm{RD}(5), \mathrm{ZB}(1), \mathrm{BQ}(2), \mathrm{GD}(4), \mathrm{JD}(3), \mathrm{KB}(3) \text {, } \\
\text { PL (5), SB (2), SR (2) }\end{array}$ \\
\hline Hap-5 & 1 & DQ (1) \\
\hline Hap-6 & 1 & JL (1) \\
\hline Hap-7 & 1 & LW (1) \\
\hline Hap-8 & 2 & LW (2) \\
\hline Hap-9 & 4 & LZ (4) \\
\hline Hap-10 & 1 & GD (1) \\
\hline Hap-11 & 1 & $\mathrm{LZ}(1)$ \\
\hline Hap-12 & 2 & $\mathrm{ZB}(2)$ \\
\hline Hap-13 & 242 & $\begin{array}{l}\text { NR (33), SS (8), SR (4), SB (4), PL (4), KB (7), JL (8), JD (6), GD (3), BQ (7), ZB (28), } \\
\text { RD (42), CN (19), LW (11), DQ (32), LZ (26) }\end{array}$ \\
\hline Hap-14 & 1 & $\mathrm{RD}(1)$ \\
\hline Hap-15 & 1 & NR (1) \\
\hline Hap-16 & 1 & SB (1) \\
\hline Hap-17 & 1 & SB (1) \\
\hline Hap-18 & 1 & $\mathrm{JL}(1)$ \\
\hline Hap-19 & 1 & PL (1) \\
\hline Hap-20 & 1 & SB (1) \\
\hline Hap-21 & 1 & SS (1) \\
\hline Hap-22 & 2 & LZ (2) \\
\hline Hap-23 & 1 & LZ (1) \\
\hline Hap-24 & 1 & SB (1) \\
\hline Hap-25 & 3 & LW (2), NR (1) \\
\hline Hap-26 & 1 & DQ (1) \\
\hline
\end{tabular}

\section{Population classification}

The average genetic distance between the populations of Tibetan yaks was 0.001452 (Table 4). The genetic distance between Kangbu and Cuona yaks was the smallest (0.000523), while the genetic distance between Sibu and Dingqing yaks was the largest (0.002912). 
Table 3. Haplotype diversity and nucleotide diversity of different Tibetan yak populations.

\begin{tabular}{lccc}
\hline Populations & Number of haplotypes & Haplotype diversity & Nucleotide diversity \\
\hline Sibu yak & 6 & 0.844 & 0.00219 \\
Riduo yak & 4 & 0.315 & 0.00057 \\
Leiwuqi yak & 6 & 0.750 & 0.00194 \\
Dingqing yak & 5 & 0.546 & 0.00347 \\
Jiangda yak & 3 & 0.600 & 0.00162 \\
Sangri yak & 3 & 0.711 & 0.00182 \\
Cuona yak & 2 & 0.409 & 0.00052 \\
Longzi yak & 7 & 0.674 & 0.00150 \\
Pali yak & 3 & 0.644 & 0.00097 \\
Sangsang yak & 3 & 0.378 & 0.00128 \\
Kangbu yak & 2 & 0.467 & 0.00060 \\
Zhongba yak & 4 & 0.383 & 0.00084 \\
Jiali yak & 3 & 0.378 & 0.00051 \\
Baqing yak & 3 & 0.582 & 0.00126 \\
Nierong yak & 5 & 0.529 & 0.00119 \\
Gongbujiangda yak & 4 & 0.778 & 0.00185 \\
Mean & 3.75 & 0.562 & 0.00138 \\
\hline
\end{tabular}

Table 4. Kimura 2-parameter genetic distance for Tibetan yak populations.

\begin{tabular}{|c|c|c|c|c|c|c|c|c|c|c|c|c|c|c|c|}
\hline Groups & $\mathrm{LZ}$ & JD & DQ & LW & NR & $\mathrm{RD}$ & ZB & BQ & GD & SR & SS & $\mathrm{CN}$ & $\mathrm{KB}$ & PL & SB \\
\hline JD & 1.570 & & & & & & & & & & & & & & \\
\hline DQ & 2.570 & 2.577 & & & & & & & & & & & & & \\
\hline LW & 1.741 & 1.876 & 2.851 & & & & & & & & & & & & \\
\hline NR & 1.334 & 1.393 & 2.397 & 1.583 & & & & & & & & & & & \\
\hline RD & 1.077 & 1.096 & 2.109 & 1.408 & 0.906 & & & & & & & & & & \\
\hline ZB & 1.181 & 1.270 & 2.254 & 1.459 & 1.018 & 0.716 & & & & & & & & & \\
\hline BQ & 1.326 & 1.366 & 2.383 & 1.578 & 1.160 & 0.901 & 1.021 & & & & & & & & \\
\hline GD & 1.695 & 1.644 & 2.715 & 1.937 & 1.520 & 1.296 & 1.447 & 1.482 & & & & & & & \\
\hline SR & 1.687 & 1.824 & 2.812 & 1.798 & 1.536 & 1.414 & 1.448 & 1.517 & 1.824 & & & & & & \\
\hline SS & 1.407 & 1.491 & 2.468 & 1.711 & 1.243 & 0.914 & 1.035 & 1.249 & 1.695 & 1.721 & & & & & \\
\hline $\mathrm{CN}$ & 1.125 & 1.038 & 2.105 & 1.495 & 0.942 & 0.579 & 0.790 & 0.920 & 1.224 & 1.491 & 0.988 & & & & \\
\hline $\mathrm{KB}$ & 1.155 & 1.054 & 2.129 & 1.525 & 0.970 & 0.611 & 0.827 & 0.945 & 1.232 & 1.515 & 1.027 & 0.523 & & & \\
\hline PL & 1.477 & 1.285 & 2.406 & 1.849 & 1.284 & 0.946 & 1.198 & 1.237 & 1.412 & 1.798 & 1.413 & 0.769 & 0.769 & & \\
\hline SB & 1.910 & 1.875 & 2.912 & 2.245 & 1.730 & 1.391 & 1.569 & 1.715 & 2.054 & 2.234 & 1.772 & 1.362 & 1.386 & 1.655 & \\
\hline JL & 1.121 & 1.156 & 2.151 & 1.489 & 0.950 & 0.558 & 0.720 & 0.957 & 1.411 & 1.540 & 0.889 & 0.602 & 0.641 & 1.026 & 1.411 \\
\hline
\end{tabular}

Data in the table must be multiplied by $10^{-3}$.

A neighbor-joining phylogenetic tree was constructed using Kimura 2-parameter genetic distances (Figure 1 and Table 4). The results showed that Tibetan yak populations were divided into 2 major groups; the Dingqing yak was in 1 group, while the other yaks clustered in another group. Two subgroups were created between the 15 groups (Figure 1). The Leiwuqi, Sangri Gongbujiangda, Baqing, Longzi, Nierong, Zhongba, Riduo, Sangsang, and Jiali populations clustered into 1 subgroup. The Sibu, Jiangda, Cuona, Kangbu, and Pali populations clustered into another subgroup.

\section{DISCUSSION}

\section{Genetic diversity of mtDNA COIII of Tibetan yak populations}

Studies examining genetic diversity can reveal the origin, genetic variation, and evo- 


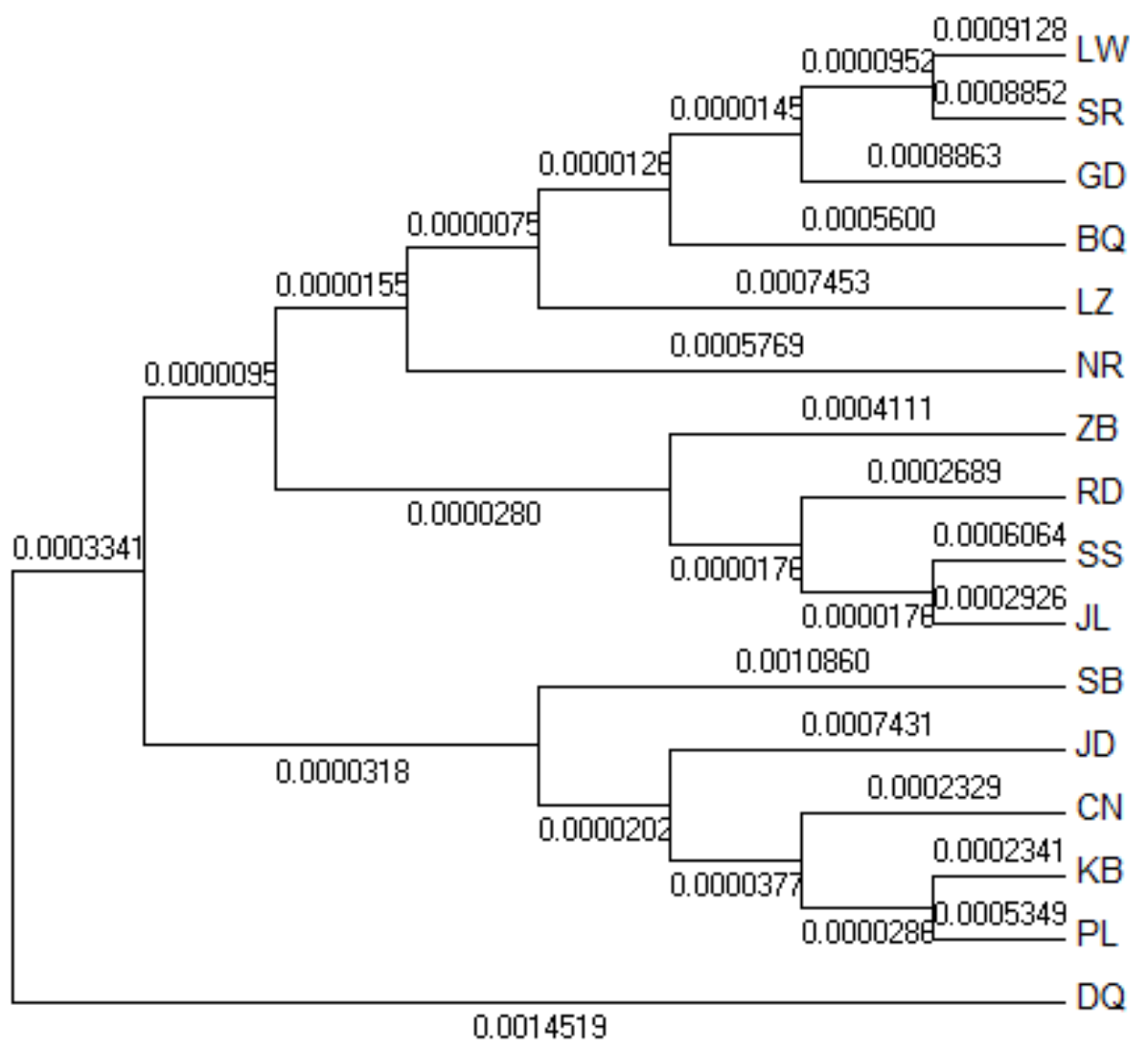

Figure 1. Neighbor-joining phylogenetic tree of Tibetan yak populations based on COIII gene sequences.

lution of a species (Zheng et al., 2012). Nucleotide and haplotype diversities are 2 important indicators used for measuring genetic variation of mtDNA within a population (Zhou et al., 2006). In our study, the average haplotype diversity among the 16 yak populations was 0.562 , while the average nucleotide diversity was 0.00138 . Although the genetic diversity of mtDNA COIII observed in our study was high, it was lower than that reported by Yang et al. (2009), Chai et al. (2011), Zhang et al. (2012), and Ji et al. (2012). However, our results were similar to those obtained using sequence-related amplified polymorphism marker analysis of $11 \mathrm{Ti}$ betan yak populations (Zhao et al., 2012). The differences observed in genetic diversity may be associated with the conservation of different genes used in various studies. The abundant genetic diversity observed in the Sibu yak population was similar to that observed by Guo et al. (2008). However, the genetic diversity of the Riduo yak population was lower. The abundant genetic diversity of Tibetan yaks plays an important role in the sustainable development of the Tibetan yak industry. It may also serve as a valuable genetic resource for yak breeding (Zhong et al., 2011). In summary, the development, effective utilization, and protection of genetic diversity of Tibetan yaks have become increasingly important for the development of the Tibetan yak industry. 


\section{Population classification}

Over the past few years, previous studies have examined the phylogenetic relationships between Tibetan yak populations (Zhao et al., 2011; Zhang et al., 2012; Ji et al., 2012). However, we observed some differences from previous reports, which is primarily because of the different research methods and populations used. Ji et al. (2012) studied the relationships between Tibetan yak populations and then divided the 11 yak populations into 5 categories: Pali, Jiangda, Baqing, Sangri, and Leiwuqi yak types. However, Zhao et al. (2011) indicated that Tibetan yak populations could be divided into 3 categories, Pali, Baqing, and Sibu yak types. Moreover, Zhang et al. (2012) suggested that Tibetan yak populations could be divided into 2 categories, with the Kangbu and Jiali yaks as 1 cluster, and the remaining populations as another cluster. In our study, cluster analysis showed that 16 Tibetan yak populations could be divided into 2 categories. Dingqing yak comprised 1 category, while the remaining populations formed another category. This is consistent with the results of microsatellite DNA marker analysis for Tibetan yak populations (Li et al., 2013). The genetic distance between Kangbu and Cuona yaks was the lowest in this study (0.000523), indicating a relatively close genetic relationship between the 2 populations. The genetic distance between Sibu and Dingqing yaks was the largest (0.002912), indicating a relatively remote genetic relationship. The genetic diversity, genetic distance, and cluster analyses of Tibetan yak populations were not directly correlated to geographical distribution. Corroborating the views of Zhang et al. (2012), Zhao et al. (2012), and Guo et al. (2008), the data indicate that the effect of the geographical environment on genetic relationships between populations has gradually weakened.

\section{ACKNOWLEDGMENTS}

Research supported by the National Science and Technology Program of China (\#2012BAD03B02) and the Genetics Discipline Program of Southwest University for Nationalities (\#2015XWD-S071007).

\section{REFERENCES}

Boore JL, Lavrov DV and Brown WM (1998). Gene translocation links insects and crustaceans. Nature 392: 667-668. Chai ZX, Zhao SJ, Ji QM, Zhang CF, et al. (2011). Study on genetic variation in Tibetan yak by RAPD technique. Acta Vet. Zootech. Sin. 42: 1380-1386.

Delarbre C, Spruyt N, Delmarre C, Gallut C, et al. (1998). The complete nucleotide sequence of the mitochondrial DNA of the dogfish, Scyliorhinus canicula. Genetics 150: 331-344.

Gissi C, Iannelli F and Pesole G (2008). Evolution of the mitochondrial genome of Metazoa as exemplified by comparison of congeneric species. Heredity 101: 301-320.

Griffiths CS (1998). The correlation of protein structure and evolution of a protein-coding gene: phylogenetic inference using cytochrome oxidase III. Mol. Biol. Evol. 15: 1337-1345.

Guo SC, Qi DL, Chen GH, Xu SX, et al. (2008). Genetic diversity and classification of domestic yaks inferred from mitochondrial DNA sequences. Acta Ecol. Sin. 28: 4286-4294.

Huang H, Li F, Alvarez RA, Ash JD, et al. (2004). Downregulation of ATP synthase subunit-6, cytochrome c oxidase-III, and NADH dehydrogenase-3 by bright cyclic light in the rat retina. Invest. Ophthalmol. Vis. Sci. 45: 2489-2496.

Ji QM, Tang YT, Zhang CF, Chai ZX, et al. (2012). Genetic diversity and evolution relationship of tibet yaks inferred from mtDNA cyt b. Acta Vet. Zootech. Sin. 43: 1723-1732.

Lavrov DV and Lang BF (2005). Poriferan mtDNA and animal phylogeny based on mitochondrial gene arrangements. Syst. Biol. 54: 651-659.

Li D, Chai ZX, Ji QM, Zhang CF, et al. (2013). Genetic diversity of DNA microsatellite for Tibetan yak. Yi Chuan 35: 
175-184.

Mipam TD, Wen Y, Fu C, Li S, et al. (2012). Maternal phylogeny of a newly found yak population in China. Int. J. Mol. Sci. 13: 11455-11470.

Pridgeon JW, Becnel JJ, Clark GG and Linthicum KJ (2009). Permethrin induces overexpression of cytochrome c oxidase subunit 3 in Aedes aegypti. J. Med. Entomol. 46: 810-819.

Rozas J, Sánchez-DelBarrio JC, Messeguer X and Rozas R (2003). DnaSP, DNA polymorphism analyses by the coalescent and other methods. Bioinformatics 19: 2496-2497.

Tamura K, Dudley J, Nei M and Kumar S (2007). MEGA4: Molecular Evolutionary Genetics Analysis (MEGA) software version 4.0. Mol. Biol. Evol. 24: 1596-1599.

Thompson JD, Gibson TJ, Plewniak F, Jeanmougin F, et al. (1997). The Clustal_X windows interface: flexible strategies for multiple sequence alignment aided by quality analysis tools. Nucleic Acid Res. 25: 4876-4882.

Tiranti V, Corona P, Greco M, Taanman JW, et al. (2000). A novel frame shift mutation of the mtDNA COIII gene leads to impaired assembly of cytochrome c oxidase in a patient affected by Leigh-like syndrome. Hum. Mol. Genet. 9: 2733-2742.

Wiener G, Han JL and Long RJ (2003). The Yak. 2nd edn. Bangkok, Thailand: Regional Office for Asia and the Pacific of the Food and Agriculture Organization of the United Nations. RAP Publication, Bangkok.

Yang WY, Chen XM, Zhong JC, Chen SM, et al. (2009). Sequence analysis and evolution relationship of Cytb gene in Bovidae. China Herbivores 29: 8-12.

Zhang CF (2012). Study on Superovulation of Tibetan Yak. Chinese Academy of Agricultural Siences Dissertation, Beijing.

Zhang CF, Xu LJ, Ji QM, Xin JW, et al. (2012). Genetic diversity and evolution relationship on mtDNA D-loop in Tibetan yaks. Acta Ecol. Sin. 32: 1387-1395.

Zhao SJ, Chen ZH, Ji QM, Chai ZX, et al. (2011). Sequence analysis of mtDNA COIII of Tibetan yaks. Sci. Agric. Sin. 44: 4902-4910.

Zhao SJ, Zhong JC, Chai ZX, Zhang CF, et al. (2012). Analysis of genetic diversity and classification of Tibetan yak breeds by SRAP molecular markers. Biotechnol. Bull. 6: 77-82.

Zheng WJ, Lai YH, Zhu SH, You XY, et al. (2012). Genetic diversity of Pseudosciaena polyactis in Zhoushan based on mitochondrial DNA D-loop region sequences. Dongwuxue Yanjiu 33: 329-336.

Zhong JC, Chai ZX, Ji QM, Zhang SJ, et al. (2011). Genetic diversity and phylogenetic analysis in Tibetan yaks. J. Southwest Nat. Coll. (Nat. Sci. Edn.) 37: 368-378.

Zhou H, Li DQ, Zhang YG, Yi XR, et al. (2006). Study on mitochondrial DNA genetic diversity of Tibetan antelope. $Y i$ Chuan 28: 299-305. 